\title{
Evolutionary analyses of KCNQI and HERG voltage-gated potassium channel sequences reveal location-specific susceptibility and augmented chemical severities of arrhythmogenic mutations Heather A Jackson and Eric A Accili*
}

\author{
Address: Department of Cellular and Physiological Sciences, Faculty of Medicine, University of British Columbia, Vancouver, BC, V6T 1Z3, Canada \\ Email: Heather A Jackson - hajacks@interchange.ubc.ca; Eric A Accili* - eaaccili@interchange.ubc.ca \\ * Corresponding author
}

Published: 30 June 2008

BMC Evolutionary Biology 2008, 8:188 doi:10.1 |86/|47|-2|48-8-188

This article is available from: http://www.biomedcentral.com//47I-2/48/8//88

(C) 2008 Jackson and Accili; licensee BioMed Central Ltd.

This is an Open Access article distributed under the terms of the Creative Commons Attribution License (http://creativecommons.org/licenses/by/2.0), which permits unrestricted use, distribution, and reproduction in any medium, provided the original work is properly cited.

\begin{abstract}
Background: Mutations in HERG and KCNQI potassium channels have been associated with Long QT syndrome and atrial fibrillation, and more recently with sudden infant death syndrome and sudden unexplained death. In other proteins, disease-associated amino acid mutations have been analyzed according to the chemical severity of the changes and the locations of the altered amino acids according to their conservation over metazoan evolution. Here, we present the first such analysis of arrhythmia-associated mutations (AAMs) in the HERG and KCNQI potassium channels.
\end{abstract}

Results: Using evolutionary analyses, AAMs in HERG and KCNQI were preferentially found at evolutionarily conserved sites and unevenly distributed among functionally conserved domains. Non-synonymous single nucleotide polymorphisms (nsSNPs) are under-represented at evolutionarily conserved sites in HERG, but distribute randomly in KCNQI. AAMs are chemically more severe, according to Grantham's Scale, than changes observed in evolution and their severity correlates with the expected chemical severity of the involved codon. Expected chemical severity of a given amino acid also correlates with its relative contribution to arrhythmias. At evolutionarily variable sites, the chemical severity of the changes is also correlated with the expected chemical severity of the involved codon.

Conclusion: Unlike nsSNPs, AAMs preferentially locate to evolutionarily conserved, and functionally important, sites and regions within HERG and KCNQI, and are chemically more severe than changes which occur in evolution. Expected chemical severity may contribute to the overrepresentation of certain residues in AAMs, as well as to evolutionary change.

\section{Background}

Two voltage-gated potassium ion channel genes, KCNQ1 and KCNH2 (HERG), encode for channels that underlie the slowly- and rapidly-activated delayed rectifier potassium currents $\left(\mathrm{I}_{\mathrm{Ks}}\right.$ and $\left.\mathrm{I}_{\mathrm{Kr}}\right)$, respectively [1-4]. Efflux of potassium ions through these channels is critical for repo- larization of the cardiac action potential. Mutations that disrupt normal biosynthesis and function of KCNQ1 and HERG have been associated with three cardiac arrhythmias: Short QT syndrome (SQTS) [5-7], atrial fibrillation $[8,9]$ and Long QT syndrome (LQTS) $[10,11]$. To date, there are close to 200 reported arrhythmia-associated 
mutations (AAMs) in each channel, with more than 95\% of which are linked to LQTS. This arrhythmia, which affects an estimated 1 in 5000-10000 people worldwide, is characterized by a prolongation of the QT interval on an electrocardiogram and can lead to Torsade de Pointes (TdP), ventricular fibrillation and sudden cardiac death $[12,13]$. More recently, the progression from LQTS into TdP has been proposed as a cause of sudden infant death syndrome (SIDS) [14-16] and sudden unexplained death syndrome (SUDS) [17]. Ninety percent of all known LQTS-associated mutations occur in HERG and KCNQ1.

In studies of larger groups of proteins, or individual proteins other than HERG and KCNQ1, disease-associated amino acid mutations (DAMs) have been analyzed according to the chemical severity of the change, as determined from Grantham's Scale [18], and the location and/ or context of the altered amino acid [19]. An up to twofold increase in clinically observable disease occurs in parallel with increases in the amount of chemical change [20]. In many proteins, DAMs are chemically more severe than changes over the course of evolution (called interspecific changes) and polymorphic changes $[21,22]$. In rhodopsin, the chemical severity of DAMs also correlates with the expected chemical severity of a given codon [21], as determined by comparison with the normally occurring human codon.

The importance of an amino acid to protein function can be inferred from its conservation over the course of metazoan evolution. In many proteins, DAMs are overabundant at evolutionarily conserved and slowly evolving sites [21-24], presumably because sites that have experienced little interspecific variation are critical for function. Mutations at these sites are likely deleterious and would be removed from the population by natural selection, if given enough time. In some proteins, DAMs are unevenly distributed among functionally conserved domains [25], even after accounting for the length and number of evolutionarily conserved amino acids. These data imply that functionally conserved domains, like conserved sites, are less tolerant to mutations because of their greater importance to the overall protein function. However, according to the Neutral Theory of Molecular Evolution, most nucleotide substitutions are phenotypically neutral and avoid natural selection [26]. Most nucleotide changes, except for disease-associated changes, might then be expected to distribute randomly throughout a protein. In a study of a large number of proteins, synonymous single nucleotide polymorphisms (sSNPs) were shown to distribute randomly consistent with a neutral phenotype [23]. Initial studies of non-synonymous (ns) SNPs in the cystic fibrosis transmembrane regulator and tuberous sclerosis complex 2 gene, were also shown to distribute randomly [22]. However, a more recent, study, using many proteins, showed that nsSNPs preferentially locate at variable sites and sites with high evolutionary rates, and are underrepresented at sites that are evolutionarily conserved and have low evolutionary rates [23]. This discrepancy may be due to differences in the nature of the nsSNPs available e.g. ns SNPs are less disruptive when located at conserved sites in some proteins versus others, or to a difference in the nature of the conserved sites e.g. conserved sites in some proteins are less tolerant to amino acid changes.

In this study, we take advantage of the $>200$ mutations reported per channel to quantitatively analyze, for the first time, the distribution of AAMs and non-synonymous SNPs within HERG and KCNQ1 and to determine the chemical severity of these changes, as compared to interspecific changes.

\section{Methods \\ Sequence alignment}

Human protein and mRNA sequences for HERG and KCNQ1 were obtained from NCBI. Other vertebrate sequences were collected from a BLASTP [27] search at ENSEMBL (default parameters) and the NCBI non-redundant database. To eliminate the inclusion of sequences from closely related paralogs, a manual reciprocal best-hit analysis was used. Sequences were aligned by ClustalX [28] using default settings. Less conserved regions in the $\mathrm{N}$ and/or C-termini were removed, leaving sequences corresponding to residues 1-906 and 83-599 of human HERG and human KCNQ1, respectively.

\section{Collection of arrhythmia-associated mutations (AAMs) and non-synonymous polymorphisms (nsSNPs)}

HERG and KCNQ1 AAMs were obtained from the NCBI OMIM database, the Human Gene Mutation Database (HGMD), the European Society of Cardiology Working Group on Arrhythmias (WGA) LQTS gene database [29] and from primary literature $[15,17,30-32]$. Only missense mutations that fell within the aligned region were used, and each was included only once, to eliminate frequency bias, but multiple disease mutations could be associated with a single site. nsSNPs were collected from NCBI dbSNP, HAPMAP international and primary literature and were assumed to be phenotypically neutral based on their inclusion in the different databases. nsSNPs that overlapped with identified disease mutations were removed. The final data set included 172 AAMs and 16 nsSNPs for HERG, and 174 AAMs (Table 1) and 12 nsSNPs for KCNQ1 (see Additional file 1).

\section{Phylogenetic analysis and determination of interspecific variability}

Aligned protein sequences, with gaps removed, were used to construct a phylogenetic tree using the maximum likelihood analysis in PHYLIP [33]. SEQBOOT, PROML, and 
Table I: Breakdown of clinical phenotype of disease mutations included in analyses

\begin{tabular}{lll}
\hline & HERG & KCNQI \\
\hline Long QT & 166 & 169 \\
Short QT & 1 & 1 \\
Atrial Fibrillation & 0 & 1 \\
SIDS & 3 & 2 \\
Long QT/SIDS & 2 & 1 \\
\hline Total & 172 & 174 \\
\hline
\end{tabular}

CONSENSE programs were used with a JTT model of evolution. Ancestral sequences were determined using the maximum likelihood method from PAMLv3.15 [34] under a Poisson model of amino acid evolution. Discrete values for interspecific variability ( 0 through $n$ ) were determined for each residue in the protein from differences between the ancestral and descendent sequences throughout the provided tree.

\section{Association between AAMs or nsSNPs and evolutionarily conserved sites}

To determine whether AAMs and nsSNPs are preferentially associated with evolutionarily conserved sites, we compared the number of observed mutations in HERG and KCNQ1 to the number of mutations expected at each site in both proteins based on neutral substitution [22]. Sites were binned according to counts of interspecific variability ( 0 through i) which were determined using PAML. The expected number of mutations was determined using the following equation

$$
\mathrm{D}_{\mathrm{i}}^{\text {expected }}=\mathrm{D}^{\text {total }} \times \mathrm{N}_{\mathrm{i}} / \mathrm{N}
$$

where $D_{i}$ expected is the expected number of mutations at sites that have undergone i substitutions, $\mathrm{D}^{\text {total }}$ is the total number of disease mutations for each channel given by $\Sigma \mathrm{D}_{\mathrm{i}}{ }^{\text {observed, }}, \mathrm{N}_{\mathrm{i}}$ is the observed number of sites in the alignment that have undergone i substitutions and $\mathrm{N}$ is the total number of residues in the gene being examined. Therefore, $\mathrm{N}_{\mathrm{i}} / \mathrm{N}$ is the fraction of sites in the gene that belong to a particular variability class (i), and if disease mutations distribute randomly throughout the protein, then $D_{i}{ }_{i}^{\text {expected }}$ will be proportional to the fraction of the total sites and the total number of disease mutations observed in each gene.

To determine whether differences in the distribution pattern between observed values and those expected from neutral theory were significant, the $\mathrm{X}^{2}$ statistic was calculated [22] and compared to a critical value for the given degrees of freedom (i-1) using the following equation,

$$
\mathrm{X}^{2}=\Sigma_{0}{ }^{\mathrm{i}}\left(\mathrm{D}^{\text {observed_-Dexpected }}\right)^{2} / \mathrm{D}^{\text {expected }}
$$

\section{Determination of codon evolutionary rate of change}

The evolutionary rates of change for codons were estimated using the maximum likelihood method implemented in the CODEML program of PAML [34,23], using a discrete gamma model (eight categories). The shape parameter was either fixed or free to vary and a likelihood ratio test was performed to evaluate model fitting. Evolutionary rates based on a Poisson model of evolution were established for every site and normalized to the maximum rate observed for each protein. Values between 0 and 1 were binned into eight different categories and used to represent eight different levels of evolutionary change. The analysis was performed on nucleotide sequence alignments of core regions, with gaps removed, of human and three closely related vertebrate orthologs, guided by protein sequence alignments. The expected number of mutations at the codons belonging to each rate category was calculated using a modification of Eq. 1 where $N_{i}$ is the number of codons belonging to i category, $\mathrm{N}$ is the total number of amino acid positions, and $\mathrm{D}^{\text {total }}$ is the total number of disease mutations for each protein used in the analysis.

\section{Distribution of AAMs among functionally important regions of the channels}

To quantify over- or underrepresentation of AAMs in functionally conserved regions of the channels, we compared their distribution to that expected from a uniform or evolutionary hypothesis [25]. First, we tested whether AAMs were distributed uniformly across the protein. The expected number of mutations in a given region was determined using the following equation

$$
D_{j}^{\text {expected }}=\left(R_{j} / R\right) \times D^{\text {total }}
$$

where $D_{j}$ expected is the expected number of disease mutations in a particular region, $\mathrm{j}, \mathrm{R}_{\mathrm{j}}$ is the number of residues found in region $\mathrm{j}, \mathrm{R}$ is the total number of residues used in the analysis $\left(\Sigma \mathrm{R}_{\mathrm{j}}\right)$ and $\mathrm{D}^{\text {total }}=\Sigma \mathrm{D}_{\mathrm{j}}$ observed or the total number of disease mutations used in the analysis.

The $\mathrm{X}^{2}$ statistic was calculated and compared to a critical value for the given degrees of freedom equal to $j-1$, where $\mathrm{j}$ is the number of different regions in the channel being analyzed, using the following equation,

$$
\mathrm{X}^{2}=\Sigma_{\mathrm{j}}\left(\mathrm{D}^{\text {observed_-Dexpected }}\right)^{2} / \mathrm{D}^{\text {expected }}
$$

Second, we tested whether the distribution of AAMs in different regions was related to the distribution of evolutionarily conserved sites. If AAMs are overrepresented at conserved sites, the number of AAMs for a given region will be proportional to the number of conserved sites 
found within that region. The expected number of mutations per region was determined using the following equation,

$$
\mathrm{D}_{\mathrm{j}}^{\text {expected }}=\Sigma_{0} \mathrm{i}\left(\left(\mathrm{a}_{\mathrm{i} j} / \mathrm{a}_{\mathrm{i}}\right) \times \mathrm{D}_{\mathrm{i}} \text { observed }\right)
$$

where $a_{i}$ is the total number of sites in a protein belonging to variability class $\mathrm{i}, \mathrm{a}_{\mathrm{ij}}$ is the number of sites of variability class $i$ found within the region $j$, and $D_{i}$ observed is the total number of disease mutations found at variability sites, $i$, across the entire protein.

\section{Chemical severity of amino acid changes}

The interspecific chemical severity of a given site was determined by the average severity (according to Grantham's Scale [18]) of all ancestor-descendent amino acid differences at that site throughout the tree, as reported by PAML. Only those interspecific changes that result from a single point mutation were included and each type of amino acid change at a given site was counted once to account for common ancestry [22]. The expected chemical severity at each site in HERG and KCNQ1 was determined by computing the average severity of all nonsynonymous changes produced by a single point mutation from the human reference codon [21].

\section{Weighted average for amino acid expected chemical severity}

To examine the involvement of specific amino acid residues in disease, the proportion of AAMs at a particular amino acid was calculated as percentage of the total AAMs in both channels. To determine whether these findings were due to an overrepresentation of certain amino acids in the proteins, data was normalized for the total number of codons for a particular residue. Finally, the weighted average for amino acid expected chemical severity was calculated by the sum of the average of each of the individual expected codon chemical severities of the residue multiplied by its contribution to the total number of codons for the residue in the two channels combined.

\section{Results}

\section{Channel Structure and Mutation Mapping}

HERG and KCNQ1 channels are likely formed by the tetrameric assembly of individual alpha subunits, each of which is composed of six transmembrane segments and cytosolic N- and C-termini (Figure 1a, b). The voltage sensing domain (VSD) is composed of the first four transmembrane segments and a pore region is composed of S5, a re-entrant P-loop containing the selectivity filter, and S6. Mapping AAMs onto the sequences and predicted topologies of HERG and KCNQ1 subunits yielded some common distribution patterns as well as some unique to the individual proteins. The final data set used for HERG consists of 172 AAMs at 134 sites and 16 nsSNPs at 16 indi- vidual sites (see Additional file 1). Of 30 sites harboring multiple AAMs, 24 sites had two, 4 sites had three and 2 sites had four. For KCNQ1, the final data set includes 174 AAMs mapping to 130 sites and 12 nsSNPs to 12 individual sites (see Additional file 1). Thirty-six sites had multiple AAMs associated with them: 30 sites with two, 5 with three and 1 with five (Figure 1).

Between the two channels, some similarities exist in the distribution patterns of AAMs in HERG and KCNQ1, supporting results gathered when much fewer mutations were known. [35]. For example, both channels contain a large number of mutations within the pore region $(23 \%$ and 29\% for HERG and KCNQ1, respectively) (Table 2). On the other hand, differences in percentages of AAMs do exist in the intra- and extra-cellular linker regions as well as the two cytosolic termini. In HERG, 23\% of all known disease mutations are found in the extracellular linkers (between S1 and S2, S3 and S4, S5 and the P-loop, and between the P-loop and S6). In KCNQ1, only 6\% are found extracellularly whereas $20 \%$ are found in the intracellular linkers (between S2-S3 and S4-S5). These differences suggest that different linker regions contribution to overall function may be channel specific. Another difference occurs in the distal termini. In HERG, $27 \%$ of disease mutations are located in the $\mathrm{N}$-terminus, compared to only $2 \%$ in KCNQ 1 . However, three quarters of these are located in the PAS (Per-Arnt-Sim) domain, a basic helixloop-helix domain that is unique to the HERG N-terminus and regulates channel closing [36].

nsSNPs distribute differently from AAMs in HERG and KCNQ1 (Figure 1). In HERG, nsSNPs are more commonly found in the cytosolic regions (15/16) compared to the transmembrane regions $(1 / 16)$. In KCNQ1, 8/12 nsSNPs are found in the cytosolic regions and 4/12 nsSNPs are found in the transmembrane region. Most of these occur at sites that do not have associated disease mutations, suggesting that location is an important determinant of disease. In both channels, however, two sites situated in cytosolic regions harbor both disease and polymorphic mutations, suggesting that amino acid identity plays a role in channel dysfunction at certain sites.

\section{AAMs occur preferentially at sites conserved throughout vertebrate evolution and at those with lower evolutionary rates of change}

The majority of AAMs mapped to sites completely conserved throughout the evolution of the respective channel: $146 / 172$ mutations $(84.9 \%)$ in HERG, mapping to 114 / $906(12.6 \%)$ of the sites used in the analysis, and 153/174 (87.9\%) in KCNQ1, mapping to 110/517 (21.3\%) of the sites used in the analysis. Conversely, 22/172 AAMs in HERG and 20/174 AAMs in KCNQ1, map to sites with interspecific variation (Figure 1). Only two sites in HERG 


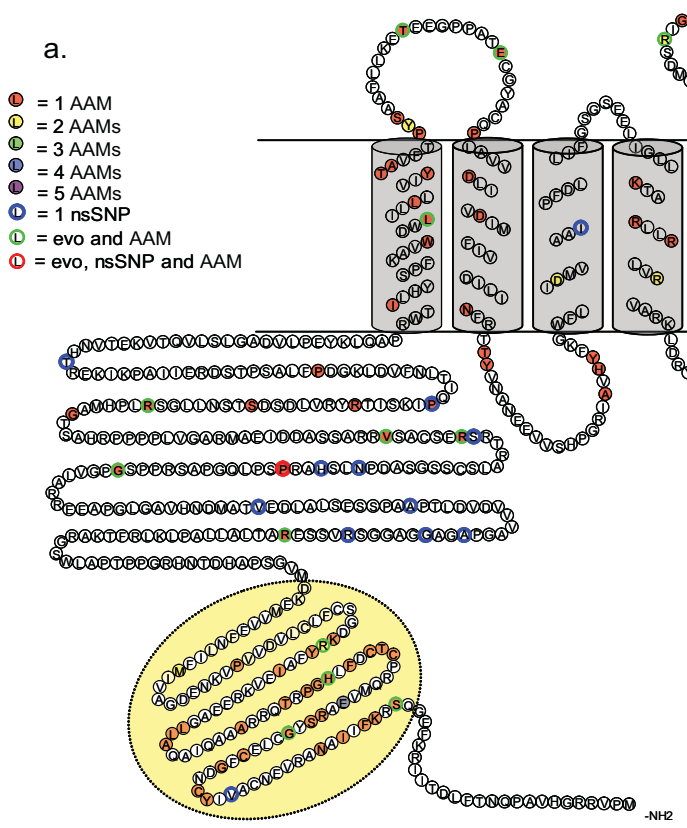

PAS Domain

b.

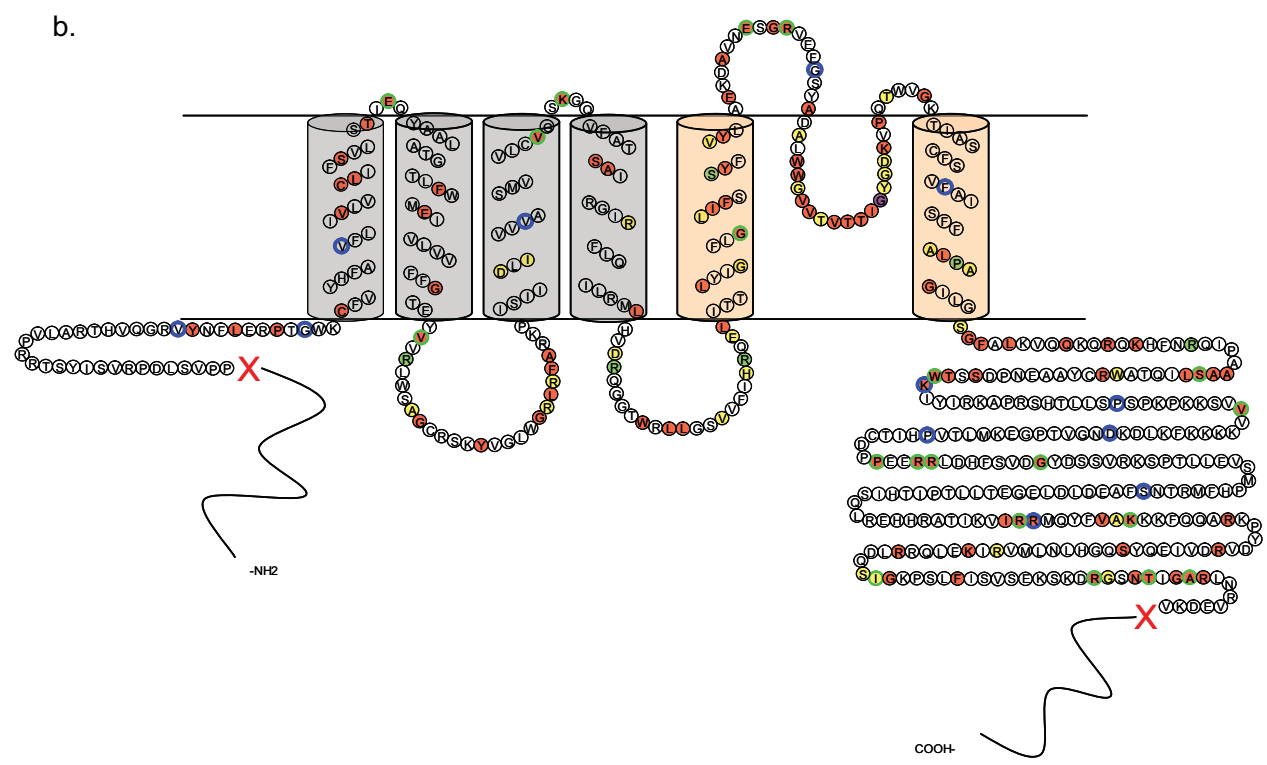

\section{Figure I}

Location of arrhythmia-associated mutations (AAMs) and non-synonymous single nucleotide polymorphisms (nsSNPs) in human HERG and KCNQI subunits. a) Schematic of the human HERG subunit. Included are I72 AAMs at I 34 sites and I6 nsSNPs at I6 sites. b) Schematic of KCNQI subunit. Included are I74 disease mutations at I30 sites and I2 nsSNPs at I 2 sites. Blue region = voltage sensing domain (SI-S4), pink region = pore forming domain (S5-S6), yellow = PAS domain or CNBD domain in cytosolic regions of the channel. Red X's delimit the region used in analysis. Circles: red = I $\mathrm{AAMs}$, yellow $=2$ mutations $/ \mathrm{site}$, green $=3$ mutations $/ \mathrm{site}$, blue $=4$ mutations $/ \mathrm{site}$, purple $=5$ mutations $/ \mathrm{site}$, blue outline $=\mathrm{I}$ $\mathrm{nsSNP} / \mathrm{site}$, green outline = overlap of disease mutation and evolutionary change, red outline = overlap of AAM, evolutionary change and nsSNP. 
Table 2: Disease mutation distribution by channel region

\begin{tabular}{lll}
\hline Region & HERG & KCNQI \\
\hline N-term & $6 \%$ & $2 \%$ \\
PAS & $21 \%$ & NA \\
VSD & $9 \%$ & $11 \%$ \\
ICL & $3 \%$ & $20 \%$ \\
ECL & $23 \%$ & $6 \%$ \\
Pore & $23 \%$ & $29 \%$ \\
C-term & $15 \%$ & $32 \%$ \\
\hline
\end{tabular}

PAS $=$ Per-Arnt-Sim domain in HERG, $\mathrm{N}$-term $=\mathrm{N}$-terminal region used in analysis (excluding PAS domain in HERG), ECL = extracellular linker regions between SI-S2, S3-S4, S5-P loop and P-loop-S6, ICL = intracellular linker regions between S2-S3 and S4-S5, VSD = voltage sensing domain (only transmembrane portions), Pore $=$ pore region including S5, P-loop and S6, C-term = C-terminal portion of channels used in this analysis.

and one in KCNQ1 involve an amino acid change that is observed in both the AAM and in interspecific change. Because the amino acid sequences of HERG and KCNQ1 are highly conserved (over 65\% complete identity between fish and human sequences in both channels for the regions used), a neutral mutational process would still produce a large number of mutations at conserved sites.

To determine whether AAMs and nsSNPs occur preferentially at evolutionarily diverse sites in HERG and KCNQ1, we utilized a quantitative approach developed previously [23]. The evolutionary relationships and the number of interspecific changes at each site were determined (Figure 2 ) and in both channels, the largest number of interspecific changes observed at any site was five. Therefore variability data was binned into six categories, ranging from completely conserved sites (0) to highly variable (5).

A greater proportion of AAMs are found at evolutionarily conserved sites, and a smaller proportion are found at variable sites, than would be expected by an underlying neutral process (Figure $3 \mathrm{a}$ ). Using $\mathrm{X}^{2}$ analysis, the difference between the distributions of observed and expected disease mutations were statistically significant in both channels, even when only the numbers of disease harboring sites were analyzed (ruling out an effect of multiple mutations at highly mutable sites) or when data were pooled to account for low numbers of expected disease mutations in higher variability classes (data not shown). In KCNQ1, nsSNPs distribute randomly but, in HERG, they were significantly underrepresented at completely conserved sites and overabundant at variable positions (Figure $3 \mathrm{~b}$ ).

To ascertain directly whether AAMs associate preferentially with sites that experience low rates of evolutionary change, we utilized an approach similar to the site analysis used above together with codon evolutionary rate obtained from CODEML in PAML [23]. For both channels, AAMs are found at sites with lower evolutionary rates a.

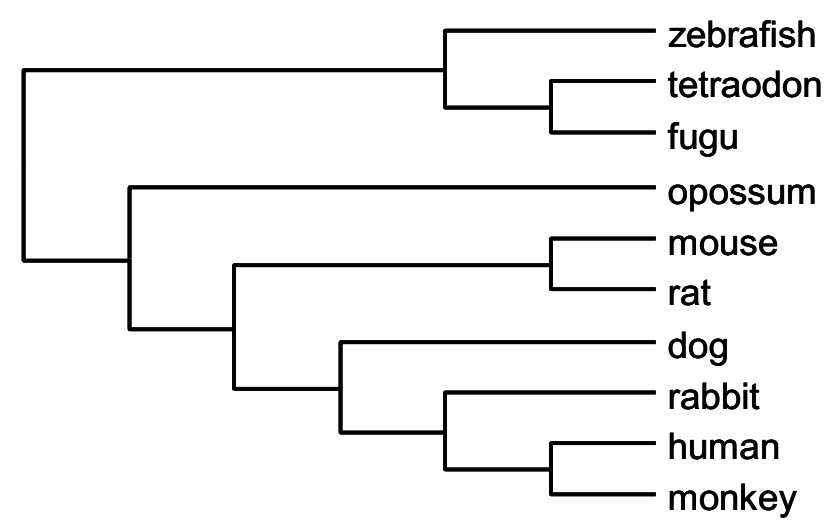

b.

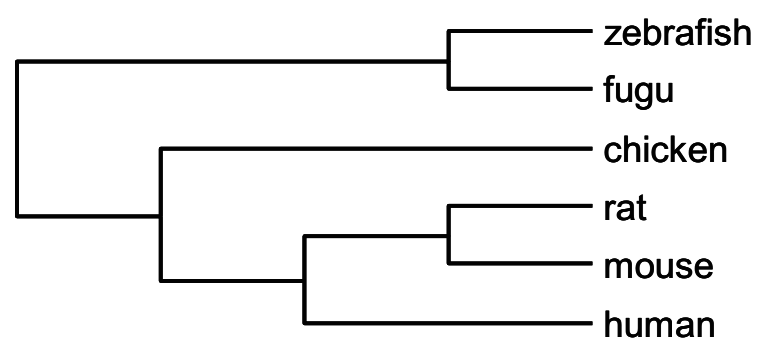

Figure 2

Cladograms of vertebrate HERG and KCNQI protein sequences. a) HERG: GenBank accession numbers/ Ensembl identifiers for protein sequences used: human (NP 000229.I), dog (NP 001003 | 45. I), zebrafish (NP 998002), monkey (ENSMMUP00000026900), rabbit (Q8WNY2), mouse (NP 038597.I), rat (NP 446401.I), opossum (ENSMODP0000000465I), fugu (NEWSINFRUP00000|6/6/5), tetraodon (GSTENP000278II00I). b) KCNQI: GenBank accession numbers/Ensembl identifiers for KCNQI protein sequences used: human (NP 000209.2), mouse (NP 032460.2), rat (NP I| 4462.I), chicken (ENSGALP000000 I 044I), fugu (NEWSINFRUP00000 I43259), zebrafish (ENSDARP0000007795I). See methods for details.

(Figure 3c). In both channels, certain AAMs were located at codons belonging to the $1^{\text {st }}$ and $2^{\text {nd }}$ evolutionary rate categories as well as to variability classes 0,1 and 2. This implies that, when AAMs are found at variable sites, they may preferentially occur at those with low evolutionary rates.

\section{Disease mutations are not equally distributed among functional regions of the channels}

Because both channels possess functional regions that are well conserved among voltage-gated channels, we tested for uneven domain distribution of AAMs. KCNQ1 and 
a.

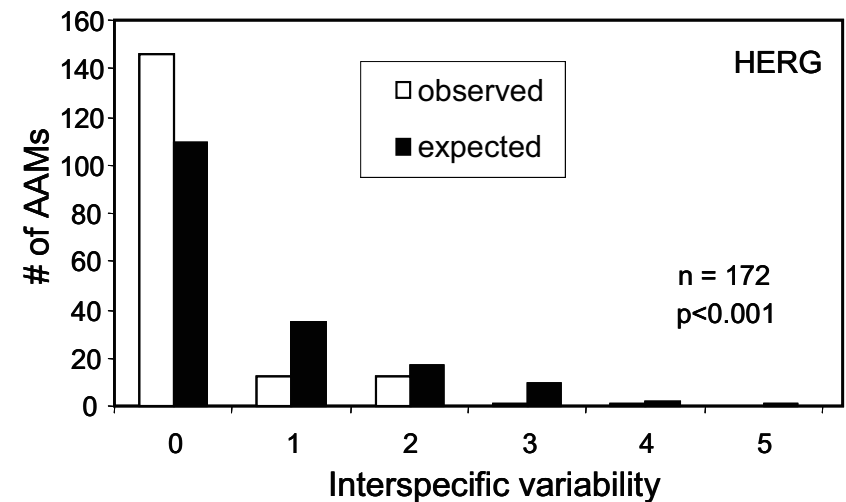

b.

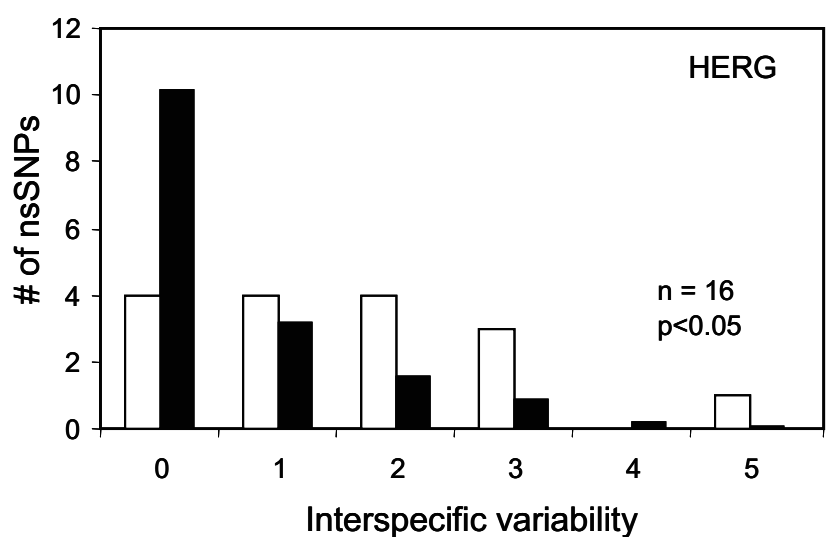

C.

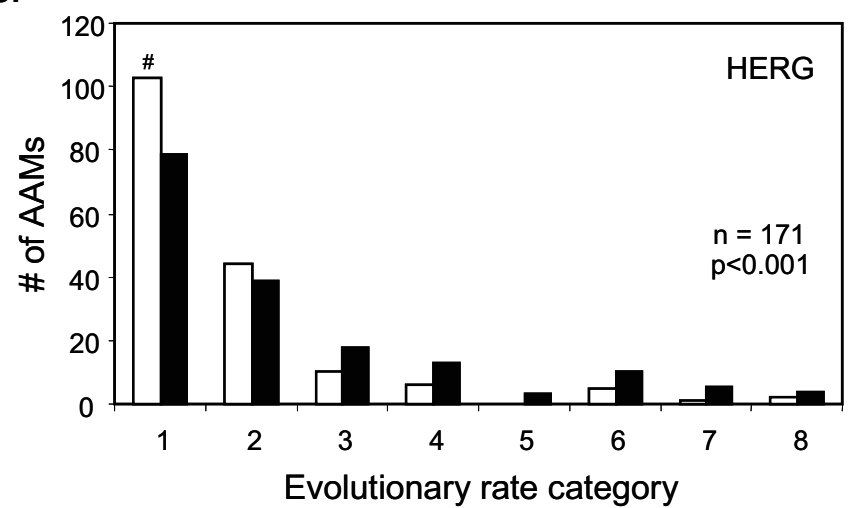

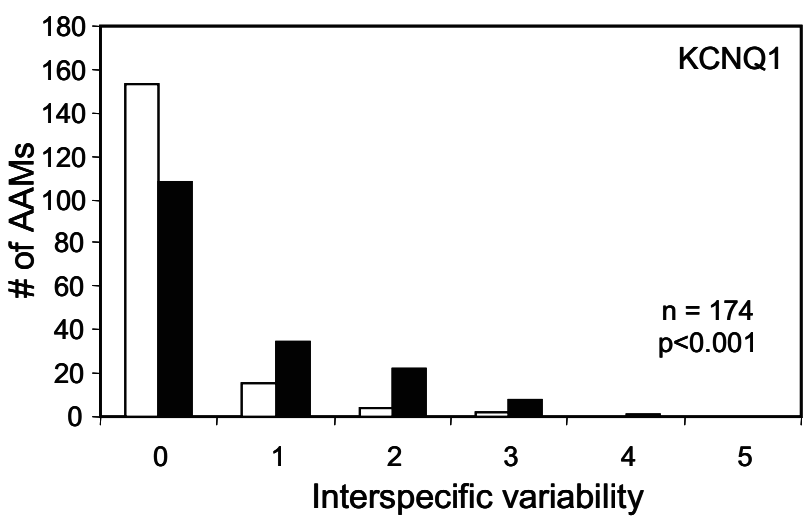
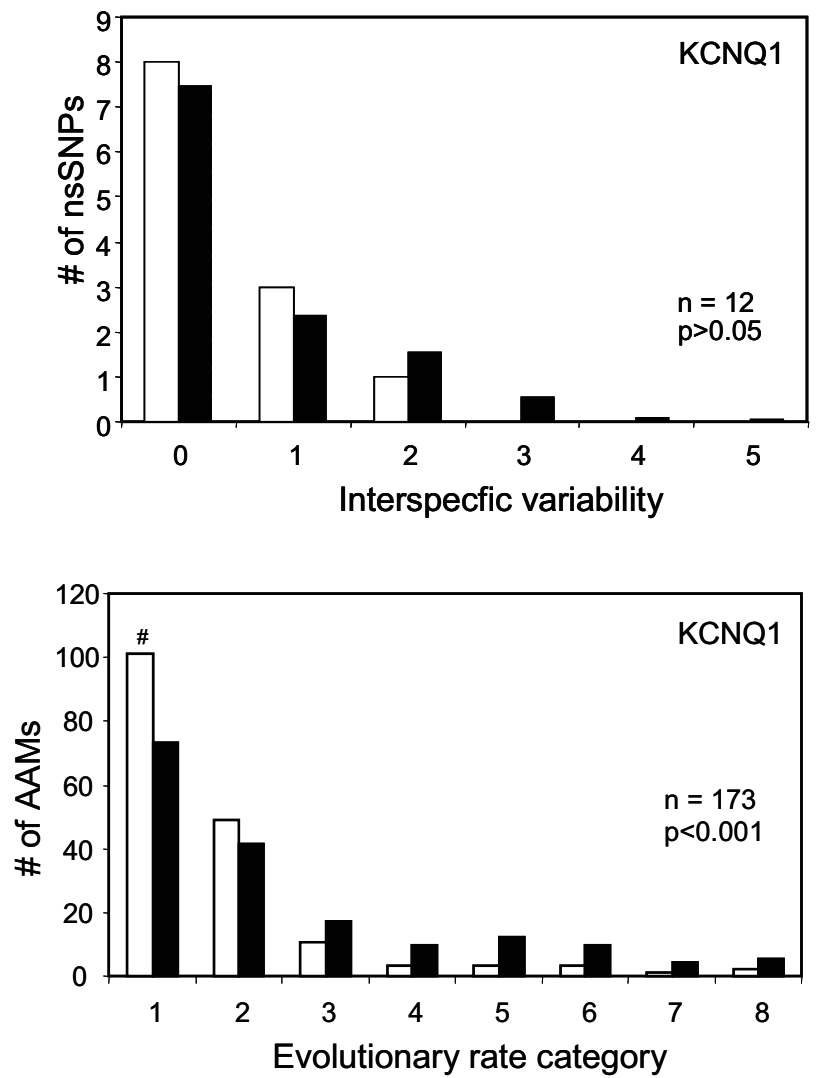

Figure 3

Arrhythmia-associated mutations in HERG and KCNQI are overrepresented at evolutionarily conserved and slowly evolving sites. a) Counts of observed (white bars) and expected (black bars) numbers of AAMs at amino acid sites in HERG and KCNQI which have undergone different numbers of substitutions among species (see methods). Because of a low number of expected mutations in the more variable positions, the $X^{2}$ statistic was also calculated for pooled data with two bins, 0 and $\mathrm{I}+$, with I degree of freedom. The number of disease mutations observed at completely conserved sites ( 0 -class) in both HERG and KCNQI is significantly higher than by chance alone: HERG, $X^{2}(5$ df $)=37.4 I, p<0.001$ or $X^{2}{ }_{(I}$ df $)=34.65, p<$ $0.00 \mathrm{I}$; KCNQI, $\mathrm{X}_{(5 \mathrm{df})}=50.45$, $\mathrm{p}<0.00 \mathrm{I}$ or $\mathrm{X}^{2}{ }_{(\mathrm{I} \text { df }}=49.37$, $\mathrm{p}<0.00 \mathrm{I}$. b) Counts of observed and expected numbers of nonsynonymous single nucleotide polymorphisms (nsSNPs). In HERG, fewer nsSNPs occur at completely conserved sites than expected by chance alone $\left(X^{2}{ }_{(5 \mathrm{df})}=22.94, \mathrm{p}<0.00 \mathrm{I}\right.$ or $\left.\mathrm{X}^{2}{ }_{(\mathrm{I} \text { df }}=10.07, \mathrm{p}<0.05\right)$ whereas in KCNQI, the distribution is not significantly different from the expected count of neutral variation $\left(X^{2}{ }_{(5 \mathrm{df})}=1.04, \mathrm{p}>0.05\right)$. $\left.c\right)$ Data were pooled to account for low numbers of expected AAMs at variable sites and significance was confirmed. The distribution of AAMs was significantly different than what would be expected by random chance for both HERG $\left(X^{2}(7 \mathrm{df})=26.10, p<0.001\right.$ or $X^{2}(1 \mathrm{df})=14.17, p<$ $0.00 \mathrm{I})$ and KCNQI $\left(X^{2}(7 \mathrm{df})=34.74, p<0.00 \mathrm{I}\right.$ or $X^{2}(\mathrm{I}$ df $\left.)=18.15, \mathrm{p}<0.00 \mathrm{I}\right)$. 
HERG were divided into six or seven regions, respectively: N-terminus, PAS domain (HERG only), VSD (S1 through S4 transmembrane regions only), pore region (excluding outer turret), extracellular linkers, intracellular linkers and the C-terminus (see Figure 1). Based on the $\mathrm{X}^{2}$ analysis, AAMs in both channels are unevenly distributed among the defined functional domains and, in general, do not support either a uniform pattern (in which the number of randomly occurring mutations are proportional to the total number of residues) or evolutionary pattern (in which the number of randomly occurring mutations are proportional to the total number of conserved residues) (Figure 4a). For both channels, AAMs are overrepresented in the pore region. AAMs are found preferentially in the intracellular (IC) linker of KCNQ1 and the extracellular (EC) linker of HERG, as well as the PAS domain of HERG, but are underrepresented in the $\mathrm{N}$ - and $\mathrm{C}$-termini of both channels. This finding is especially striking for KCNQ1 considering $32 \%$ of its disease mutations are found in the C-terminus. The number of AAMs in the VSD of HERG and KCNQ1 were not different from the expected number based on a uniform or evolutionary distribution.

To examine whether the overall conservation of a region (average variability/site in domain) exerts a non-additive influence on the disease susceptibility [25], the average number of AAMs per site in a given region of HERG and KCNQ1 was plotted against its average variability per site. These values were correlated for KCNQ1, but not for HERG (Figure 4b). The slope of this relationship in KCNQ1 was greater than those based on the uniform or evolutionary hypothesis. This indicates that AAMs are overabundant in conserved regions and less than expected in variable regions, which suggests that the regional sequence conservation may play a role in KCNQ1 disease susceptibility.

\section{The chemical severities of AAMs are different than changes observed throughout evolution}

Two sites in each channel are associated with both an AAM and nsSNP. In each case, the AAM and nsSNP involve different amino acid substitutions. Additionally, of the 42 disease sites that overlap with interspecific change, only two sites in HERG and one site in KCNQ1 display an AAM that is the same as an interspecific change, suggesting that the identity of the amino acid contributes to susceptibility to disease. In previous studies, the chemical severity of disease-causing mutations, determined by the Grantham's Scale, were on average larger than interspecific changes, and not correlated with evolutionary changes found at the same sites [21,22]. The average chemical severity of AAMs in HERG and KCNQ1 were also larger than those observed throughout evolution (Table 3 ). Furthermore, we found no correlation between the chemical severities of both types of change in HERG and
KCNQ1 at variable sites that harbor a disease mutation (Figure 5a).

The chemical severity of amino acid changes may depend on the expected chemical severity of mutations that can arise from a single nucleotide substitution in the codon involved [21]. For KCNQ1 and HERG, the chemical severity of AAMs and expected chemical severity were correlated (Figure 5b). Interspecific and expected chemical severities, however, were not correlated for KCNQ1, and had a very small correlation coefficient for HERG (Figure 5c) (which becomes non-significant when three outliers are removed; not shown). These findings are consistent with previous studies, which suggest that interspecific chemical severity is not influenced by the expected chemical severity of the codon involved but rather by the process of natural selection [21]. Nonetheless, the chemical severities of changes tolerated at variable sites may be influenced by the codon's expected chemical severity. When completely conserved sites were removed (Figure $5 \mathrm{~d})$, the expected chemical severity of the involved codon was correlated with interspecific chemical severity, for both HERG and KCNQ1, but to a lesser extent than with disease chemical severity based on slope and correlation co-efficient.

We next compared the average chemical severities of AAMs and nsSNPs (Table 3). We found a significant difference between AAMs and nsSNPs in KCNQ1, but not in HERG, suggesting that other factors, such as location, may play a larger role in causing channel dysfunction for AAMs in HERG compared to KCNQ1. There were no significant differences between nsSNP and interspecific severities in either channel.

\section{Involvement of specific amino acids in arrhythmogenic disease}

We next determined which amino acids in HERG and KCNQ1 are targets in arrhythmia-causing mutations. Figure 6 displays the amino acid spectrum of residues involved in disease for both genes. We found that $28 \%$ of all AAMs in these two channels have occurred at either a glycine or arginine residue (Figure 6a). This is similar to a broader protein analysis reported previously [37]. The contribution of a particular residue to the overall disease spectrum may be influenced by the proportion of the given amino acid in these proteins. Therefore, we determined the number of disease mutations at a given residue as a proportion of the total number of the residue in both proteins (Figure 6b). Arginine and glycine residues remain highly represented suggesting they are more likely to be involved in a disease phenotype compared to other residues. The proportion of tryptophan residues involved in disease is also high, although the total number of tryptophan residues is low. 
The high involvement of some residues in disease may be due to a high mutation rate that occurs at $\mathrm{CpG}$ dinucleotides [21] which is a result of a cytosine to thymine transition. This transition is possible in triplets coding for only five of the twenty amino acids, which are: arginine (4/6 codons), serine ( $1 / 6$ codons), proline ( $1 / 4$ codons), threonine ( $1 / 4$ codons) and alanine ( $1 / 4$ codons $)$. Of the total number of AAMs in HERG and KCNQ1, only 13.3\% are due to a $\mathrm{C} / \mathrm{T}$ transition at a $\mathrm{CpG}$ dinucleotide. Of all arginine mutations resulting in disease, $47 \%$ are due to this specific nucleotide transition. These numbers suggest that CpG dinucleotides may contribute to the disease process but that this is not the only factor responsible for the high numbers of mutations in this sub-set of amino

a.

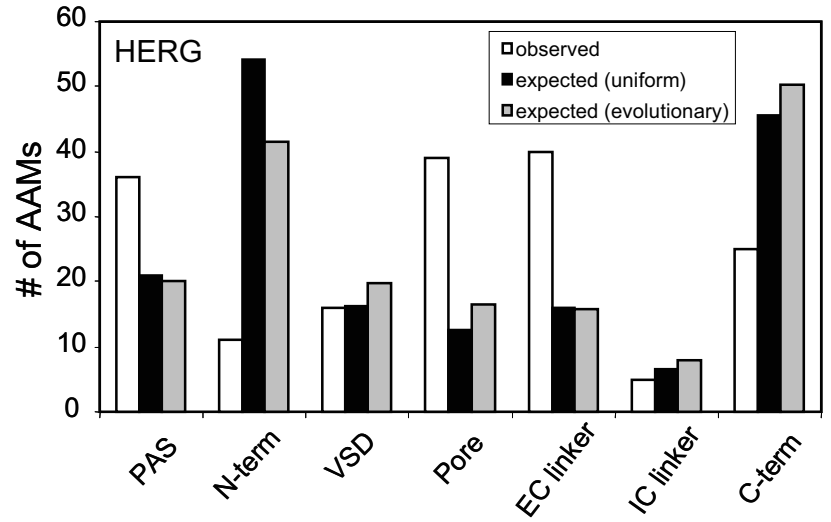

b.

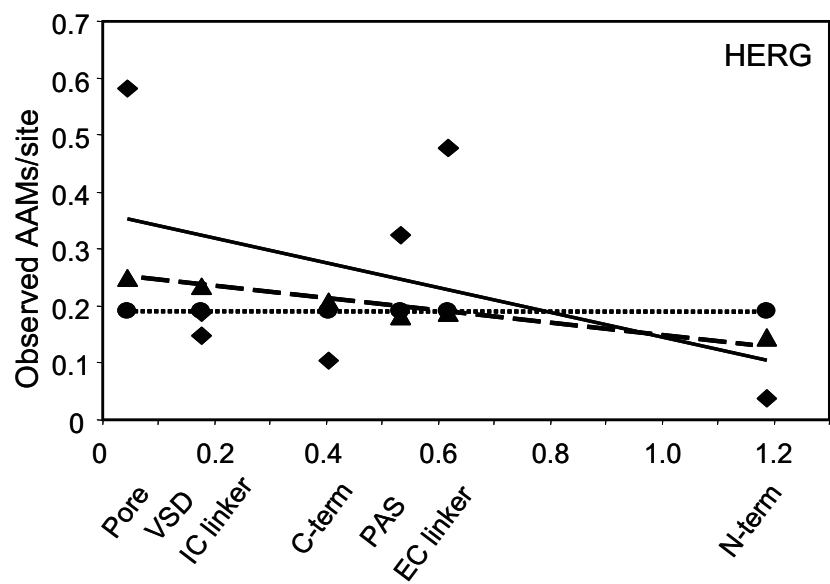

Variability/site in domain

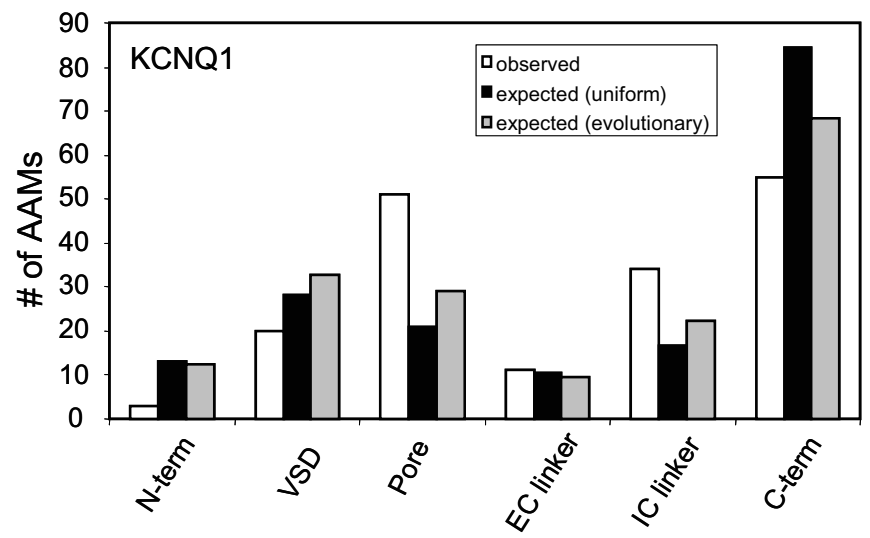

Channel region

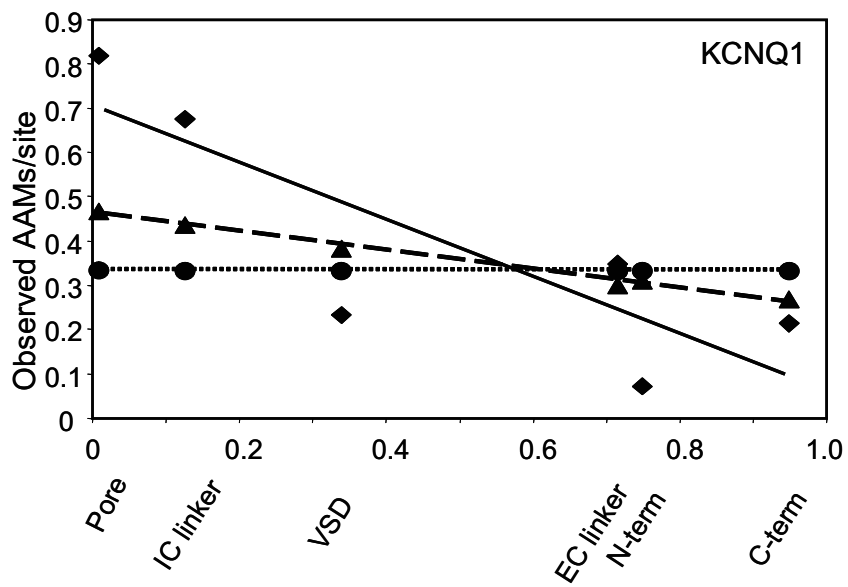

Variability/site in domain

Figure 4

Arrhythmia-associated mutations are unevenly distributed among functionally conserved regions of HERG and KCNQ I even after accounting for total length and evolutionary conservation of individual sites therein. a) Counts of observed number of AAMs per region (white), counts of expected number of AAMS based on a uniform distribution across each gene (black) and expected number of disease mutations based on an evolutionary distribution within each region (gray). Disease mutations are unevenly distributed among different regions of the channel: $\mathrm{HERG}_{\text {uniform }}\left(\mathrm{X}^{2}{ }_{(6 \mathrm{df})}=145.10, \mathrm{P}<\right.$ $0.00 \mathrm{I}), \mathrm{HERG}_{\text {evolutionary }}\left(\mathrm{X}^{2}{ }_{(6 \mathrm{df})}=\mathrm{I} \mathrm{I} 6.55, \mathrm{p}<0.00 \mathrm{I}\right), \mathrm{KCNQI}_{\text {uniform }}\left(\mathrm{X}^{2}{ }_{(5 \mathrm{df})}=8 \mathrm{I} .59, \mathrm{p}<0.00 \mathrm{I}\right)$ and $\mathrm{KCNQI}_{\text {evolutionary }}\left(\mathrm{X}^{2}(5 \mathrm{df})=\right.$ $37.39, \mathrm{p}<0.00 \mathrm{I})$. b) Scatter plots showing the relationship between channel region conservation (average variability/site within domain) and the average number of observed disease mutations per site (diamonds) or expected number of disease mutations per site based on a uniform (circles) or evolutionary (triangles) distribution. Dotted and dashed lines indicate fits for expected uniform and evolutionary distribution, respectively. Solid lines represent best-fit regression of observed data. The correlation is significant for KCNQI but not for HERG, and the best fit of the KCNQI data is significantly different from the other two hypotheses. 
acids. A role for factors other that $\mathrm{CpG}$ dinucleotide hypermutability is also supported by the high involvement of glycines and tryptophans in disease (Figure 6b), which do not possess CpG dinucleotides.

Our findings that the overall chemical severity of AAMs is greater than those of interspecific variation and SNPs, and that the chemical severity of AAMs correlates with expected chemical severity at those individual codons, suggest that chemical severity is on a continuum and that a threshold severity exists which, once crossed, results in disease. We might then expect that whether mutations at specific amino acids cause disease at all also depends on the expected chemical severity of the involved codon. To examine this, the proportion of total AAMs at a particular residue was plotted against the calculated weighted average of expected chemical severity (Figure 6c). A significant, positive correlation was found between these suggesting that expected chemical severity of a site contributes to the probability of obtaining a disease mutation, as well as to the severity of that mutation.

\section{Discussion}

In this study, we show that that AAMs are overabundant at evolutionarily conserved and slowly evolving sites, which are likely critical for channel function and thus intolerant of changes in amino acid sequence. Because KCNQ1 and HERG are highly conserved, an underlying neutral mutational process could produce large numbers of mutations at conserved sites. Our data provide the quantitative backing to support an over representation of AAMs at evolutionarily conserved positions in these channels. A smaller than expected, but still substantial, numbers of AAMs were found at sites that show interspecific variation. However, only two sites in HERG and one in KCNQ1 are converted to residues that are both an AAM and an interspecific change. Thus, the identity of the residue, rather than its location, is most responsible for producing the disease at sites that have undergone evolutionary change.

HERG and KCNQ1 possess structurally defined domains with specific functions that have been strongly preserved throughout the course of evolution [36,38]. We found that AAMs are found preferentially in some domains, even after accounting for their size and evolutionary conservation. In both channels, the pore region possesses an overabundance of mutations, while both $\mathrm{N}$ - and C-termini possess an underabundance of mutations. The extracellular linker region had an overabundance of mutations in HERG, whereas the intracellular linker had an overabundance of mutations in KCNQ1. This implies that the extracellular linker may be more important to overall function in HERG, whereas the intracellular linker is more critical for function in KCNQ1. The overabundance of mutations in the PAS domain, not present in KCNQ1, highlights its functional importance and suggests that that the addition of a functionally important domain in a protein can increase susceptibility to disease. Overall regional conservation, which takes into account the average variability per site in a domain, contributes to the uneven regional distribution of disease-causing mutations in some proteins [25], but we found this only for KCNQ1 (Figure 4b). Therefore, factors other than domain size, site conservation and regional conservation must influence the domain specific distribution of AAMs in HERG, and possibly in KCNQ1 as well.

NsSNPs are underabundant at conserved sites in HERG, but distribute randomly in KCNQ1. The latter finding may be due to a small sample size, although the numbers of nsSNPs analyzed in HERG were similarly small. This difference between the channels may be because nsSNPs are less disruptive when located at conserved sites in KCNQ1, or because conserved sites in HERG are less tolerant to amino acid changes. Nevertheless, the distribution of analyzed nsSNPs in both channels suggests that they are phenotypically neutral. The different patterns of nsSNP distribution between the KCNQ1 and HERG underscore the need to identify and quantitatively analyze the distribution of more nsSNPs on each protein, and to ascertain their impact on channel function and arrhythmia susceptibility. In HERG, it is known that some, but not all, polymorphisms may alter channel function [39], and also contribute to an increased QT interval duration [40].

In both HERG and KCNQ1, AAMs are chemically more severe than interspecific changes tolerated throughout evolution or polymorphisms that are not associated with disease. Codons with a higher expected chemical severity are associated with disease mutations with a high chemical severity. These data are in keeping with those found previously in rhodopsin [21] and suggest that the intrinsic potential of the involved codon contributes to disease chemical severity.

Table 3: Average chemical differences of amino acid changes

\begin{tabular}{|c|c|c|c|}
\hline Gene & Disease & nsSNP & Interspecific \\
\hline HERG & $\begin{array}{l}93.63(3.99) \\
\mathrm{n}=172\end{array}$ & $\begin{array}{l}75.50(10.55) \\
n=16\end{array}$ & $\begin{array}{l}67.37(1.97) * \\
\mathrm{n}=296\end{array}$ \\
\hline KCNQI & $\begin{array}{l}88.29(3.75) \\
n=174\end{array}$ & $\begin{array}{l}52.25(8.66) \dagger \\
n=12\end{array}$ & $\begin{array}{l}58.32(2.74) * \\
n=168\end{array}$ \\
\hline
\end{tabular}

$*, \dagger$ indicate a significant difference from disease severity $(\dagger \mathrm{p}<$ $0.0001,{ }^{*} p<0.008$ ) as determined by a Mann-Whitney $U$ test. There was no significant difference between the average chemical differences observed in disease vs. polymorphic changes $(p=0.218)$ for HERG. There was no significant difference between the average chemical differences observed in polymorphic and interspecific amino acid changes in either HERG $(p=0.144 I)$ or KCNQI $(p=0.6671)$. 
a.

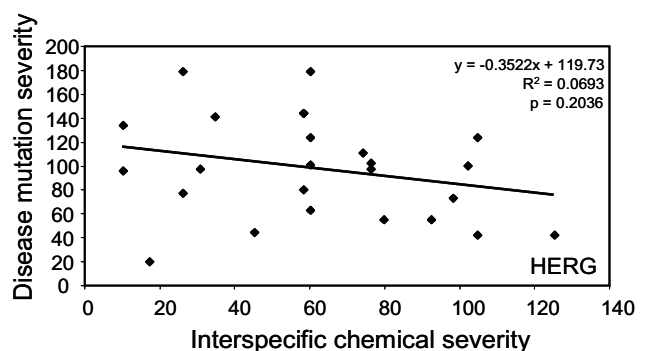

b.

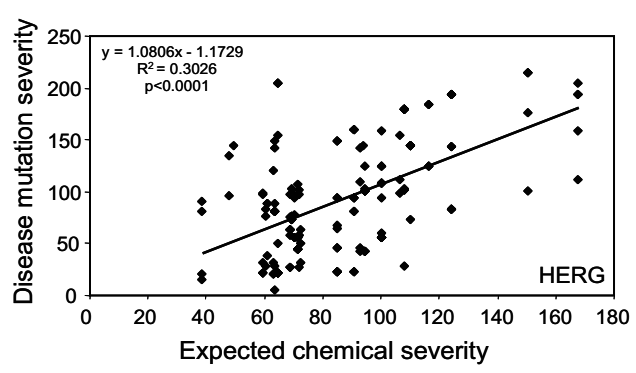

c.

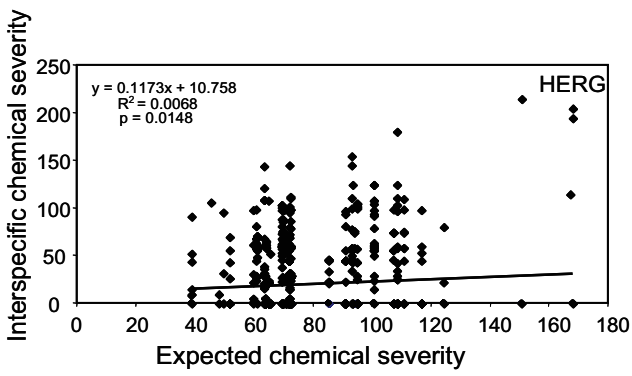

d.

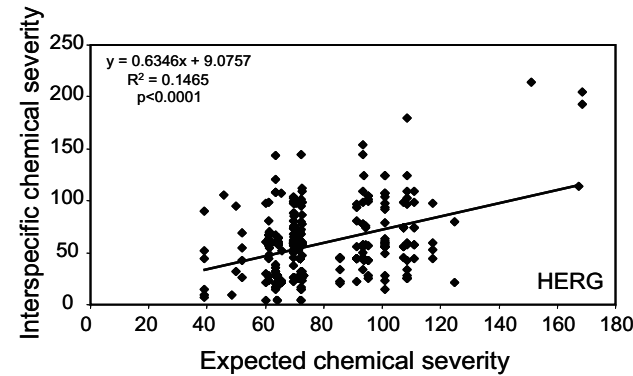

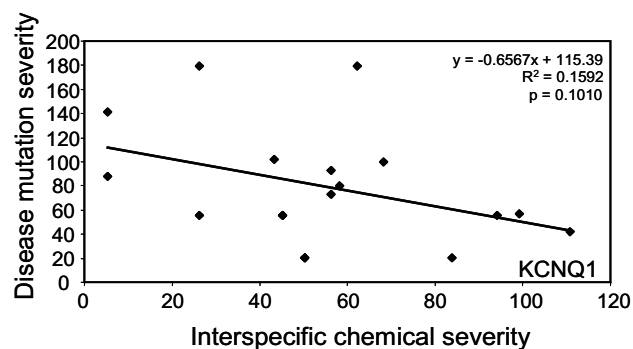
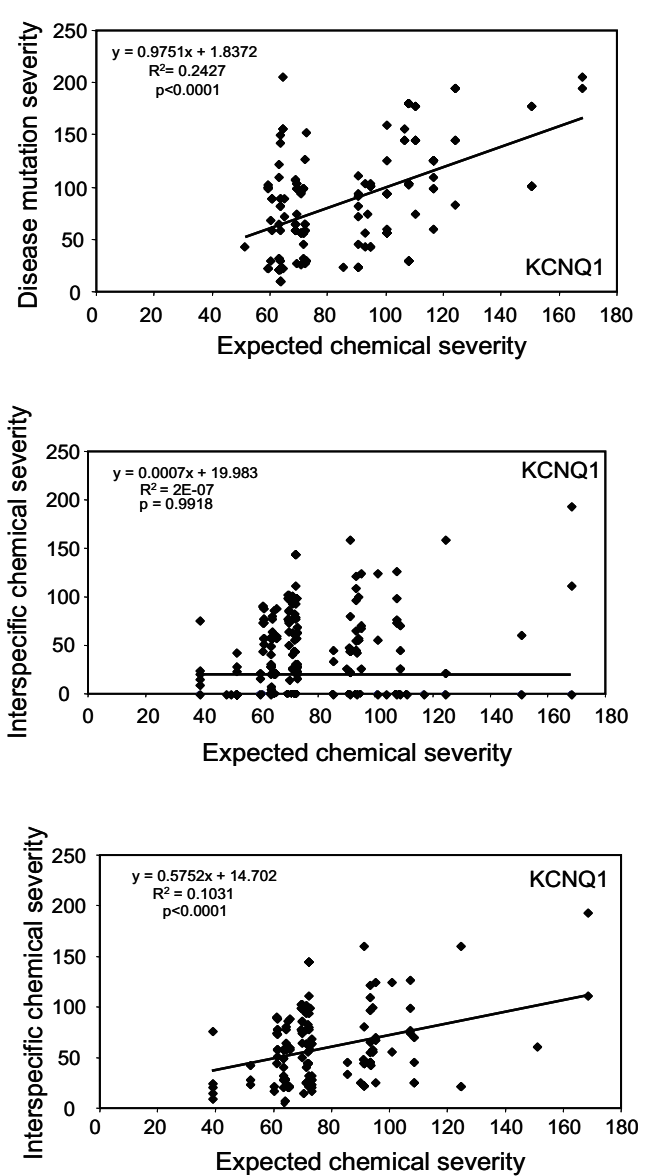

Figure 5

Arrhythmia-associated mutation and interspecific chemical severities correlate with the expected chemical severity of the reference codon in HERG and KCNQI, but not with each other. a) Plot of AAM vs. interspecific chemical severities. No significant correlation is observed for either HERG $(p=0.20)$ or KCNQI $(p=0.10)$. b) Plots of AAM vs. expected chemical severities. A significant correlation is observed for both HERG $(p<0.000 I)$ and KCNQI ( $<<0.000 \mathrm{I})$. $c)$ Plots of interspecific vs. expected chemical severities (all sites). A significant correlation is observed for HERG ( $P=0.0 \mathrm{I} 48)$ but not for KCNQI ( $p=0.9918)$. For both proteins, the correlation $(r)$ statistic, and the slopes of the regression, for interspecific vs. expected chemical severities are significantly smaller $(p<0.00 I)$ than those for AAM vs. expected chemical severities ('b', above). d) Plots of interspecific vs. expected chemical severities using only variable sites. A significant correlation is observed for both HERG $(p<0.000 \mathrm{I})$ and KCNQI $(p<0.000 \mathrm{I})$. The correlations and slopes of the linear regression are significantly larger $(p<0.00 I)$ than those using all sites ('c', above). For both proteins, the slopes of the linear regression are significantly smaller than those for AMM vs. expected chemical severities ('b', above) The correlation ( $r$ ) is significantly smaller than that for AMM vs. expected chemical severities ('b', above) for HERG $(p<0.05)$ and not for KCNQI $(p=0.058)$. Significant differences in the average chemical severity were tested using the Mann-Whitney $U$ test whereas the correlation differences between expected chemical severity and disease or interspecific severity were tested for significance using the z-score calculations. 
a.

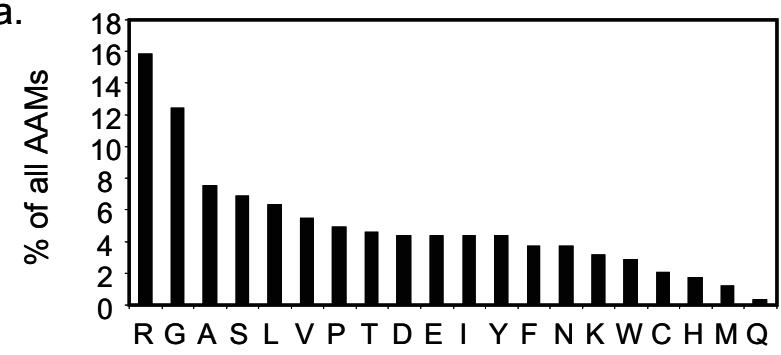

b.

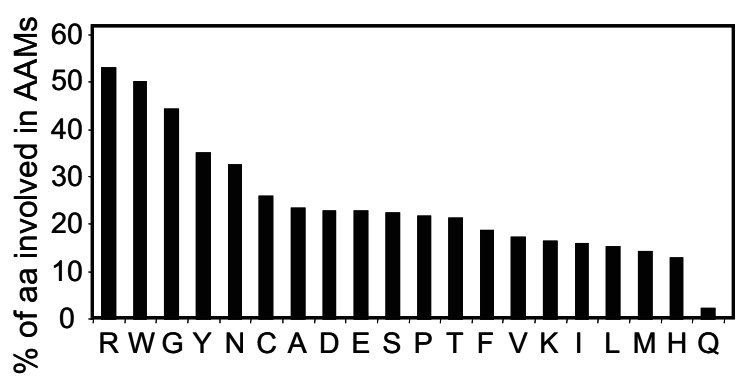

c.

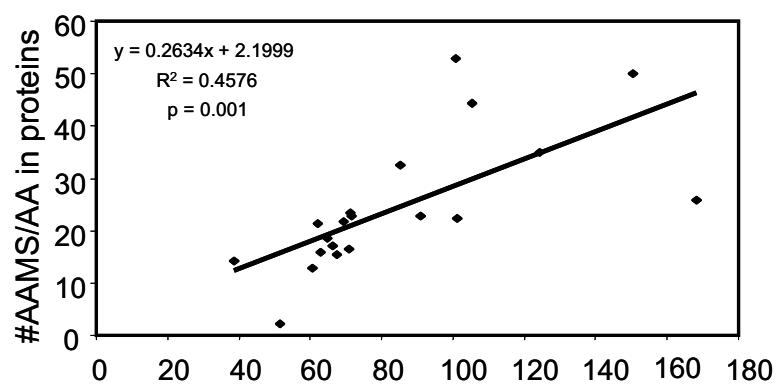

Weighted average expected chemical severity

\section{Figure 6}

Mutations occur predominantly at arginine and glycine residues. a) Percentage of AAMs that occur at a given amino acid residue. b) Relative contribution of a given amino acid residue in HERG and KCNQI to AAMs. The total number of AAMs that occur at a given amino acid was divided by the total number of residue sites in the combination of the two protein regions used in the analysis. c) The number of AAMs in KCNQI and HERG at each of the twenty residues, proportional to their occurrence in the two channels, is significantly correlated $(p=0.00 \mathrm{I})$ with the amino acid's weighted average expected chemical severity.

We also provide novel evidence that the expected chemical severity of a codon contributes to the overrepresentation of certain amino acids in HERG and KCNQ1 AAMs, especially arginine, tryptophan and glycine. In a recent study of 437 proteins, these three amino acids were also highly overrepresented in DAMs [37]. Therefore, we predict that expected chemical severity plays an important role in determining the propensity of a given codon to cause disease in many proteins, in addition to other factors such as the residue's roles in biosynthesis, function and stability of the channels. Finally, the chemical severities of interspecific changes in KCNQ1 and HERG also correlate with those expected for the codons, when only evolutionarily variable sites are considered. These novel data argue that, in addition to a predicted role of natural selection, the expected chemical severity of the codon contributes to variation observed over the course of evolution in these channels. Despite the presumed predominance of natural selection, the genetic code has been shown to influence the mutational process when evolutionary divergence is low [41]. These data are significant given the uncertainty as to the role of natural selection versus non-adaptive forces in shaping genotypic and phenotypic variation $[42,43]$.

Our analyses may be influenced by the fact that some mutations may, in a systematic way, never be detected. For example, AAMs that lead to death before natural birth, or to SUDs, may never be identified unless the fetus or victim is subsequently screened for arrhythmogenic mutations. This could, in turn, reduce the number of observed mutations unique to certain sites or functional domains. The evaluation of AAMs has broadened, and identification of mutations in KCNQ1 and HERG, and in other candidate genes, associated with SIDs and SUDs has been carried out [14-17]. Identification of AAMs in a more broad population may reveal a different sub-set of mutations that localize to unique regions within the channels, or more strongly support the susceptibility of sites and functional domains identified in this study to arrhythmogenic mutations.

\section{Conclusion}

Our study represents the first quantitative evolutionary and chemical severity analysis of AAMs in the HERG and KCNQ1 potassium channel genes. Unlike nsSNPs, AAMs preferentially locate to evolutionarily conserved, and functionally important, sites and regions within HERG and KCNQ1, and are chemically more severe than changes which occur in evolution. Expected chemical severity may contribute to the overrepresentation of certain residues in AAMs, as well as to changes observed throughout evolution.

Our findings, together with those from other studies, suggest that novel DAMs and AAMs may be recognized quickly by surveying naturally occurring variation among species [21]. If a SNP identified in an individual does not appear in other species at that position, then it is likely to be disease-causing. The location of AAMs (to conserved or variable regions and/or residues) may correlate with clinical severity or other characteristics of the diseases. In the case of Long QT syndrome, genotype and specific mutations have been shown to contribute to phenotype [4447] and the underlying genetic defects contribute to risk stratification, prevention and therapy $[12,48]$. Unfortu- 
nately, there is still considerable variation in Long QT phenotype and age of disease onset [13]. Therefore, continued discovery and mapping of mutations, as done in this study, along with parallel studies on disease phenotype will ultimately lead to a better understanding of the genotype-phenotype relationship, help to better predict the outcome of novel disease mutations and aid in development of mutation-specific therapies.

\section{Authors' contributions}

Together, HJ and EA designed the study, interpreted the data and drafted the manuscript. HJ performed the data collection and the evolutionary and statistical analyses. Both authors have read and have approved the final manuscript.

\section{Additional material}

\section{Additional file 1}

A list of AAMs and nsSNPs in KCNQ1 and HERG used in the analyses. A list of all disease mutations and SNPs which were used in these analyses along with their location within each channel, the disease phenotype with which they are associated and the database reference from which they were collected.

Click here for file

[http://www.biomedcentral.com/content/supplementary/14712148-8-188-S1.xls]

\section{Acknowledgements}

We would like to acknowledge Christian Peters for helpful feedback on the manuscript as well as James Ho, Chris Fung, Thomas Li, and Navinder Dhaliwat for preliminary work and discussion on the project. Furthermore, we would like to acknowledge three anonymous reviewers that provided helpful feedback on the manuscript.

\section{Funding Sources}

Supported by grants from the Natural Sciences and Engineering Research Council of Canada (HAJ and EAA). E.A.A. is also the recipients of a Tier II Canada Research Chair.

\section{Disclosures}

None

\section{References}

I. Sanguinetti MC, Curran ME, Zou A, Shen J, Spector PS, Atkinson DL, Keating MT: Coassembly of K(V)LQTI and minK (IsK) proteins to form cardiac I(Ks) potassium channel. Nature 1996, 384(6604):80-83.

2. Sanguinetti MC, Jiang C, Curran ME, Keating MT: A mechanistic link between an inherited and an acquired cardiac arrhythmia: HERG encodes the IKr potassium channel. Cell I995, 8 I (2):299-307.

3. Trudeau MC, Warmke JW, Ganetzky B, Robertson GA: HERG, a human inward rectifier in the voltage-gated potassium channel family. Science 1995, 269(5220):92-95.

4. Barhanin J, Lesage F, Guillemare E, Fink M, Lazdunski M, Romey G: K(V)LQTI and IsK (minK) proteins associate to form the
I(Ks) cardiac potassium current. Nature 1996 384(6604):78-80.

5. Brugada R, Hong K, Dumaine R, Cordeiro J, Gaita F, Borggrefe M, Menendez TM, Brugada J, Pollevick GD, Wolpert C, Burashnikov E, Matsuo K, Wu YS, Guerchicoff A, Bianchi F, Giustetto C, Schimpf R, Brugada P, Antzelevitch C: Sudden death associated with shortQT syndrome linked to mutations in HERG. Circulation 2004, 109(I):30-35.

6. Gaita F, Giustetto C, Bianchi F, Wolpert C, Schimpf R, Riccardi R, Grossi S, Richiardi E, Borggrefe M: Short QT Syndrome: a familial cause of sudden death. Circulation 2003, 108(8):965-970.

7. Bellocq C, van Ginneken AC, Bezzina CR, Alders M, Escande D, Mannens MM, Baro I, Wilde AA: Mutation in the KCNQI gene leading to the short QT-interval syndrome. Circulation 2004, I09(20):2394-2397.

8. Chen YH, Xu SJ, Bendahhou S, Wang XL, Wang Y, Xu WY, jin HW, Sun H, Su XY, Zhuang QN, Yang YQ, Li YB, Liu Y, Xu HJ, Li XF, Ma N, Mou CP, Chen Z, Barhanin J, Huang W: KCNQI gain-of-function mutation in familial atrial fibrillation. Science 2003, 299(5604):25I-254.

9. Hong K, Bjerregaard P, Gussak I, Brugada R: Short QT syndrome and atrial fibrillation caused by mutation in KCNH2. J Cardiovasc Electrophysiol 2005, 16(4):394-396.

10. Wang Q, Curran ME, Splawski I, Burn TC, Millholland JM, VanRaay TJ, Shen J, Timothy KW, Vincent GM, de Jager T, Schwartz PJ, Toubin JA, Moss AJ, Atkinson DL, Landes GM, Connors TD, Keating MT: Positional cloning of a novel potassium channel gene: KVLQTI mutations cause cardiac arrhythmias. Nat Genet 1996, I 2(1):17-23.

II. Curran ME, Splawski I, Timothy KW, Vincent GM, Green ED, Keating MT: A molecular basis for cardiac arrhythmia: HERG mutations cause long QT syndrome. Cell 1995, 80(5):795-803.

12. Schwartz PJ: Management of long QT syndrome. Nat Clin Pract Cardiovasc Med 2005, 2(7):346-35I.

13. Roden DM: Clinical practice. Long-QT syndrome. N EnglJ Med 2008, 358(2): 169-176.

14. Schwartz PJ, Priori SG, Bloise R, Napolitano C, Ronchetti E, Piccinini A, Goj C, Breithardt G, Schulze-Bahr E, Wedekind H, Nastoli J: Molecular diagnosis in a child with sudden infant death syndrome. Lancet 200I, 358(9290): I342-1343.

15. Arnestad M, Crotti L, Rognum TO, Insolia R, Pedrazzini M, Ferrandi C, Vege A, Wang DW, Rhodes TE, George AL Jr., Schwartz PJ: Prevalence of long-QT syndrome gene variants in sudden infant death syndrome. Circulation 2007, II 5(3):36I-367.

16. Wang DW, Desai RR, Crotti L, Arnestad M, Insolia R, Pedrazzini M, Ferrandi C, Vege A, Rognum T, Schwartz PJ, George AL Jr.: Cardiac sodium channel dysfunction in sudden infant death syndrome. Circulation 2007, I I5(3):368-376.

17. Tester DJ, Ackerman MJ: Postmortem long QT syndrome genetic testing for sudden unexplained death in the young. $J$ Am Coll Cardiol 2007, 49(2):240-246.

18. Grantham R: Amino acid difference formula to help explain protein evolution. Science 1974, I 85(4I 54):862-864.

19. Botstein $D$, Risch $N$ : Discovering genotypes underlying human phenotypes: past successes for mendelian disease, future approaches for complex disease. Nat Genet 2003, 33 Suppl:228-237.

20. Krawczak M, Ball EV, Cooper DN: Neighboring-nucleotide effects on the rates of germ-line single-base-pair substitution in human genes. Am J Hum Genet 1998, 63(2):474-488.

21. Briscoe $A D$, Gaur $C$, Kumar $S$ : The spectrum of human rhodopsin disease mutations through the lens of interspecific variation. Gene 2004, 332:107-1 18.

22. Miller MP, Kumar S: Understanding human disease mutations through the use of interspecific genetic variation. Hum Mol Genet 200I, 10(21):2319-2328.

23. Subramanian S, Kumar S: Evolutionary anatomies of positions and types of disease-associated and neutral amino acid mutations in the human genome. BMC Genomics 2006, 7:306.

24. Mooney SD, Klein TE: The functional importance of diseaseassociated mutation. BMC Bioinformatics 2002, 3:24.

25. Miller MP, Parker JD, Rissing SW, Kumar S: Quantifying the intragenic distribution of human disease mutations. Ann Hum Genet 2003, 67(Pt 6):567-579.

26. Nei M: Selectionism and neutralism in molecular evolution. Mol Biol Evol 2005, 22(I 2):23 I8-2342. 
27. Altschul SF, Gish W, Miller W, Myers EW, Lipman DJ: Basic local alignment search tool. J Mol Biol I990, 2I 5(3):403-4I0.

28. Thompson JD, Gibson TJ, Plewniak F, Jeanmougin F, Higgins DG: The CLUSTAL $X$ windows interface: flexible strategies for multiple sequence alignment aided by quality analysis tools. Nucleic Acids Res 1997, 25(24):4876-4882.

29. Inherited Arrhythmias Database [http://www.fsm.it/cardmoc/]

30. Mank-Seymour AR, Richmond JL, Wood LS, Reynolds JM, Fan YT, Warnes GR, Milos PM, Thompson JF: Association of torsades de pointes with novel and known single nucleotide polymorphisms in long QT syndrome genes. Am Heart J 2006, 152(6): $1116-1122$.

31. Aydin A, Bahring S, Dahm S, Guenther UP, Uhlmann R, Busjahn A Luft FC: Single nucleotide polymorphism map of five long-QT genes. J Mol Med 2005, 83(2): I59- 165.

32. Millat G, Chevalier P, Restier-Miron L, Da Costa A, Bouvagnet $P$, Kugener B, Fayol L, Gonzalez Armengod C, Oddou B, Chanavat V Froidefond E, Perraudin R, Rousson R, Rodriguez-Lafrasse C: Spectrum of pathogenic mutations and associated polymorphisms in a cohort of 44 unrelated patients with long QT syndrome. Clin Genet 2006, 70(3):2I 4-227.

33. Felsenstein J: Inferring phylogenies from protein sequences by parsimony, distance, and likelihood methods. Methods Enzymol 1996, 266:418-427.

34. Yang Z: PAML: a program package for phylogenetic analysis by maximum likelihood. Comput Appl Biosci 1997, I3(5):555-556.

35. Splawski I, Shen J, Timothy KW, Lehmann MH, Priori S, Robinson JL, Moss AJ, Schwartz PJ, Towbin JA, Vincent GM, Keating MT: Spectrum of mutations in long-QT syndrome genes. KVLQTI, HERG, SCN5A, KCNEI, and KCNE2. Circulation 2000, I02(I 0): I I78-I I 85

36. Sanguinetti MC, Tristani-Firouzi M: hERG potassium channels and cardiac arrhythmia. Nature 2006, 440(7083):463-469.

37. Vitkup D, Sander C, Church GM: The amino-acid mutational spectrum of human genetic disease. Genome Biol 2003, $4(\mathrm{II}):$ R72.

38. Jespersen T, Grunnet M, Olesen SP: The KCNQI potassium channel: from gene to physiological function. Physiology (Bethesda) 2005, 20:408-416.

39. Anson BD, Ackerman MJ, Tester DJ, Will ML, Delisle BP, Anderson $\mathrm{CL}$, January $\mathrm{CT}$ : Molecular and functional characterization of common polymorphisms in HERG $(\mathrm{KCNH} 2)$ potassium channels. Am J Physiol Heart Circ Physiol 2004, 286(6):H2434-4I.

40. Newton-Cheh C, Guo CY, Larson MG, Musone SL, Surti A, Camargo AL, Drake JA, Benjamin EJ, Levy D, D'Agostino RB Sr., Hirschhorn JN, O'Donnell C J: Common genetic variation in $\mathrm{KCNH} 2$ is associated with QT interval duration: the Framingham Heart Study. Circulation 2007, I | 6(10): I |28-I I36.

41. Benner SA, Cohen MA, Gonnet GH: Amino acid substitution during functionally constrained divergent evolution of protein sequences. Protein Eng 1994, 7(I I): 1323-1332.

42. Yi SV: Non-adaptive evolution of genome complexity. Bioessays 2006, 28(I0):979-982.

43. Lynch $M$ : The evolution of genetic networks by non-adaptive processes. Nat Rev Genet 2007, 8( I 0):803-8I3

44. Brink PA, Crotti L, Corfield V, Goosen A, Durrheim G, Hedley P, Heradien M, Geldenhuys G, Vanoli E, Bacchini S, Spazzolini C, Lundquist AL, Roden DM, George AL Jr., Schwartz PJ: Phenotypic variability and unusual clinical severity of congenital long- $Q T$ syndrome in a founder population. Circulation 2005, I I 2(I7):2602-2610

45. Crotti L, Spazzolini C, Schwartz PJ, Shimizu W, Denjoy I, Schulze-Bahr E, Zaklyazminskaya EV, Swan H, Ackerman MJ, Moss AJ, Wilde AA Horie M, Brink PA, Insolia R, De Ferrari GM, Crimi G: The common long-QT syndrome mutation KCNQI/A34IV causes unusually severe clinical manifestations in patients with different ethnic backgrounds: toward a mutation-specific risk stratification. Circulation 2007, I I 6(2I):2366-2375.

46. Moss AJ, Zareba W, Kaufman ES, Gartman E, Peterson DR, Benhorin J, Towbin JA, Keating MT, Priori SG, Schwartz PJ, Vincent GM, Robinson JL, Andrews ML, Feng C, Hall WJ, Medina A, Zhang L, Wang Z: Increased risk of arrhythmic events in long-QT syndrome with mutations in the pore region of the human ether-a-gogo-related gene potassium channel. Circulation 2002, 105(7):794-799.
47. Tan HL, Bardai A, Shimizu W, Moss AJ, Schulze-Bahr E, Noda T, Wilde AA: Genotype-specific onset of arrhythmias in congenital long-QT syndrome: possible therapy implications. Circulation 2006, I | 4(20):2096-2103.

48. Priori SG, Schwartz PJ, Napolitano C, Bloise R, Ronchetti E, Grillo M, Vicentini A, Spazzolini C, Nastoli J, Bottelli G, Folli R, Cappelletti D: Risk stratification in the long-QT syndrome. N Engl J Med 2003, 348(19): 1866-1874.
Publish with Biomed Central and every scientist can read your work free of charge

"BioMed Central will be the most significant development for disseminating the results of biomedical research in our lifetime. "

Sir Paul Nurse, Cancer Research UK

Your research papers will be:

- available free of charge to the entire biomedical community

- peer reviewed and published immediately upon acceptance

- cited in PubMed and archived on PubMed Central

- yours - you keep the copyright 